\title{
Repeated PSMA-targeting radioligand therapy of metastatic prostate cancer with ${ }^{131} \mathrm{I}-\mathrm{MIP}-1095$
}

\author{
Ali Afshar-Oromieh ${ }^{1,2}$ (D) Uwe Haberkorn ${ }^{1,2}$ - Christian Zechmann ${ }^{1}$ - Thomas Armor ${ }^{3}$. \\ Walter Mier $^{1}$ • Fabian Spohn ${ }^{1}$ • Nils Debus ${ }^{1}$ - Tim Holland-Letz ${ }^{4}$ John Babich ${ }^{5,6,7}$ • \\ Clemens Kratochwil ${ }^{1}$
}

Received: 16 January 2017 / Accepted: 23 February 2017 /Published online: 9 March 2017

(C) The Author(s) 2017. This article is published with open access at Springerlink.com

\begin{abstract}
Purpose Prostate-specific membrane antigen (PSMA)targeting radioligand therapy (RLT) was introduced in 2011. The first report described the antitumor and side effects of a single dose. The aim of this analysis was to evaluate toxicity and antitumor activity after single and repetitive therapies. Methods Thirty-four men with metastatic castration-resistant prostate cancer received PSMA-RLT with ${ }^{131}$ I-MIP-1095. Twenty-three patients received a second, and three patients a third dose, timed at PSA progression after an initial response to the preceding therapy. The applied doses were separated in three groups: $<3.5,3.5-5.0$ and $>5.0 \mathrm{GBq}$. Antitumor and side-effects were analyzed by blood samples and other clinical data. Follow-up was conducted for up to 5 years.
\end{abstract}

Electronic supplementary material The online version of this article (doi:10.1007/s00259-017-3665-9) contains supplementary material, which is available to authorized users.

Ali Afshar-Oromieh

a.afshar@gmx.de

1 Department of Nuclear Medicine, Heidelberg University Hospital, INF 400, 69120 Heidelberg, Germany

2 Clinical Cooperation Unit Nuclear Medicine, German Cancer Research Center, Heidelberg, Germany

3 Progenics Pharmaceuticals, Inc., New York, NY, USA

4 Department of Biostatistics, German Cancer Research Center, Heidelberg, Germany

5 Division of Radiopharmaceutical Sciences, Department of Radiology, Weill Cornell Medicine, New York, NY, USA

6 Citigroup Biomedical Imaging Center, Weill Cornell Medicine, New York, NY 10021, USA

7 Meyer Cancer Center, Weill Cornell Medicine, New York, NY 10021, USA
Results The best therapeutic effect was achieved by the first therapy. A PSA decline of $\geq 50 \%$ was achieved in $70.6 \%$ of the patients. The second and third therapies were significantly less effective. There was neither an association between the applied activity and PSA response or the time-to-progression. Hematologic toxicities were less prevalent but presented in a higher percentage of patients with increasing number of therapies. After hematologic toxicities, xerostomia was the second most frequent side effect and presented more often and with higher intensity after the second or third therapy. Conclusion The first dose of RLT with ${ }^{131}$ I-MIP-1095 presented with low side effects and could significantly reduce the tumor burden in a majority of patients. The second and third therapies were less effective and presented with more frequent and more intense side effects, especially hematologic toxicities and xerostomia.

Keywords Prostate cancer · PSMA · Prostate-specific membrane antigen $\cdot$ Endoradiotherapy

\section{Introduction}

The clinical translation of ${ }^{99 \mathrm{~m}} \mathrm{Tc}-,{ }^{18} \mathrm{~F}$ - and ${ }^{68} \mathrm{Ga}$-labeled prostate specific membrane antigen (PSMA)-targeting tracers for single-photon emission tomography (SPECT) or positron emission tomography (PET) are considered a significant step forward for the diagnostics of prostate cancer (PCa) [1-5]. The very first small-molecule PSMA ligands that presented convincing results in men were ${ }^{123}$ I-MIP-1072 and ${ }^{123}$ I-MIP1095 [6]; which were described in the literature in 2009 [7]. However, these pioneering ligands were under-recognized at their introduction. One reason might be that the mediumenergy cyclotron-produced ${ }^{123} \mathrm{I}$ is more expensive then compact cyclotron-produced ${ }^{18} \mathrm{~F}$ or generator nuclides such as 
${ }^{99 \mathrm{~m}} \mathrm{Tc}$ or ${ }^{68} \mathrm{Ga}$. On-site labeling with radioiodine can also be more complex than labeling of chelator-containing ligands.

Since internalization occurs after binding of antibodies and small-molecule ligands to PSMA, these molecules may also be good candidates for endoradiotherapy [8]. First therapy was done with the monoclonal antibody J591. In phase 1 and 2 clinical trials, J591 was radiolabeled with ${ }^{90} \mathrm{Y}$ or ${ }^{177} \mathrm{Lu}$ leading to promising early results [9-11]. However, monoclonal antibodies are large molecules which show poor permeability in solid tumors and slow clearance from the circulation. Due to their specific tumor targeting and faster pharmacokinetics in comparison to full-length antibodies, small-molecule PSMA ligands were also considered promising for systemic radioligand therapy (RLT) of metastatic prostate cancer. DOTA or DOTAG -chelator-containing ligands can easily be labeled with a variety of different diagnostic or therapeutic radionuclides, making them favorable candidates for clinical application. Finally, such compounds became available in 2013 and first clinical results were now published in 2016 [12-21]. However, the first PSMA RLTs based on a radioiodine-labeled compound were already conducted between July 2011 and June 2012 [22]. After PET-based dosimetry with ${ }^{124}$ I-MIP-1095, 28 patients received one single treatment with ${ }^{131}$ I-MIP-1095 and were followed until first PSA progression [22]. An additional eight patients received their first cycle of ${ }^{131}$ I-MIP-1095 afterwards, before PSMA RLT was switched to ${ }^{177}$ Lu-PSMA ligands.

Here, we report our long-term follow-up of 34 patients with a special focus on repeated ${ }^{131}$ I-MIP-1095 therapies, which were not timed at fixed intervals but were only scheduled after progression of disease.

\section{Materials and methods}

\section{Patients}

Between July 2011 and October 2013, 36 men with progressive metastatic castration-resistant prostate cancer (mCRPC) were referred to PSMA RLT after having received all approved therapies available at that time.

A PSMA-positive tumor phenotype was demonstrated with ${ }^{68} \mathrm{Ga}-\mathrm{PSMA}-11$ PET/CT. The characteristics of all 36 patients who received at least one therapy with ${ }^{131} \mathrm{I}$-MIP1095 are given in Table 1. Out of these, two patients were lost for follow-up. Twenty-three of the remaining patients received a second and three patients received a third therapy. Overall, 60 treatments were applied in the 34 evaluable patients. The second and third therapeutic activity was applied in case of PSA progression following an initial decline after the first therapy (supplementary figure 1).
The follow-up until first PSA progression of 28 patients receiving one single therapy has previously been published [22].

Depending on the efficiency of radiolabeling, patients received an average radioactivity of $4.2 \pm 1.4 \mathrm{GBq}$ (1.9-7.2; median 3.7) for the first therapy. The activities of the second and third therapies were also depending on labeling yields. However, if blood cell count was not completely recovered, the responsible physician could indicate individual dose reductions. For the second therapy, we applied $3.2 \pm$ 1.2 GBq (2.0-6.4; median 2.7) and for the third therapy, 2.4 $\pm 1.0 \mathrm{GBq}(1.5-3.5 ; 2.2)$. In order to evaluate possible association of side effects with therapy doses, the applied doses were separated in three groups: $<3.5 \mathrm{GBq}$ (group 1), 3.55.0 GBq (group 2) and >5.0 GBq (group 3).

On the first (prior to radioactivity administration) and on the last day of the hospitalization, blood samples were collected from all patients for the measurement of the following parameters: hematology, electrolytes, glutamic oxaloacetic transaminase, glutamate-pyruvate transaminase, gammaglutamyl transferase, alkaline phosphatase, bilirubin, urea, creatinine, glomerular filtration rate, prostate-specific antigen (PSA), thyroid-stimulating hormone, free triiodothyronine and free thyroxine.

In order to reduce possible thyroid uptake of free radioiodine, 60 drops of sodium perchlorate (Irenat ${ }^{\circledR}$, Bayer, Berlin, Germany) were given p.o. ca. $1 / 2 \mathrm{~h}$ prior to therapy and $3 \times 20$ drops in the next 14 days after therapy administration. Also, prior to therapy, the patients received 1000-2000 $\mathrm{mL}$ of $0.9 \% \mathrm{NaCl}$ solution over 1 day. The therapy solution (described below) was administered by intravenous infusion over 20-30 min.

Post administration, patients were treated as in-patients on the nuclear medicine therapy ward for 5-8 days according to German radiation protection laws. The vital parameters as well as side effects or adverse effects were recorded during the whole hospitalization time. In an attempt to to reduce therapy-induced damage of the salivary glands, the patients received five times per day lemon juice and ice packs during the first day to reduce organ perfusion over the parotids and submandibular glands for the period of their hospitalization.

Whole-body scintigraphy was acquired at the last day of the hospitalization. The patients were then followed-up further for side effects and blood parameters taken every 2 weeks over a period of 10 weeks (the thyroid parameters were controlled monthly by blood tests). After the 10th week, the frequency of the blood analyses was at the discretion of the treating oncologists. Any further (long-term) available information about the relevant medical history of the patients was collected until the death of the patients. At the time this manuscript was submitted, four patients were still living while all others have passed away. 
Table 1 Patients' characteristics

\begin{tabular}{|c|c|c|c|}
\hline & 1st Therapy & 2nd Therapy & 3rd Therapy \\
\hline Dose (GBq) & $4.2 \pm 1.4(1.9-7.2)$ & $3.2 \pm 1.2(2.0-6.4)$ & $2.4 \pm 1.0(1.5-3.5)$ \\
\hline Time to PSA progression (d) & $116 \pm 141(18-735)$ & $62 \pm 28(28-117)$ & $42 *$ \\
\hline \multirow[t]{2}{*}{ PSA baseline (ng/ml) } & $261(1.1-2109.0)$ & $212(0.3-1497.0)$ & $480(125.0-878.0)$ \\
\hline & $\begin{array}{l}\text { Chemotherapy prior } \\
\text { to PSMA therapy } \\
14 / 34(44 \%)\end{array}$ & $\begin{array}{l}\text { CRPC } \\
\text { All patients }\end{array}$ & $\begin{array}{l}\text { Age (mean, range) } \\
68(49-81)\end{array}$ \\
\hline \multicolumn{4}{|l|}{ Types of PCa } \\
\hline Local PCa (3 patients) & $\begin{array}{l}\text { Lymph node metastases } \\
\text { (18 patients) }\end{array}$ & $\begin{array}{l}\text { Visceral metastases } \\
\quad(3 \text { patients })\end{array}$ & $\begin{array}{l}\text { Bone metastases } \\
\quad(28 \text { patients })\end{array}$ \\
\hline
\end{tabular}

* Only one patient presented with PSA decline after the third cycle; all others presented with continuous progressive disease despite RLT

Hematologic toxicities were analyzed according to common terminology criteria (CTC) of the World Health Organization. Xerostomia grades were as follows: grade 0 (no toxicity), grade 1 (measurable in sctintigraphy only), grade 2 (noticeable for patients, but no treatment required), grade 3 (treatment required), grade 4 (significant impairment) and grade 5 (percutaneous endoscopic gastrostomy required). Dysgeusia, xerophthalmia and fatigue syndrome were recorded as present or absent, including their duration. Bone pain was recorded as present or absent including, its change after the therapy.

In case of biochemical relapse of $\mathrm{PCa}$, the patients were re-evaluated for an additional therapy with ${ }^{131} \mathrm{I}-$ MIP-1095. Requirements for this additional therapy were a sufficient bone marrow reserve (leucocytes $\geq 3000 / \mu 1$ blood; platelets $\geq 75,000 / \mu \mathrm{l}$ blood) and a response to the prior therapy shown by PSA decline and/or morphological response demonstrated by computed tomography (CT), magnetic resonance imaging (MRI) or positron emission Tomography (PET) with ${ }^{68}$ Ga-PSMA-11.

\section{Radiopharmaceutical}

The radioiodinated compound ${ }^{131} \mathrm{I}-\mathrm{MIP}-1095$ was prepared as described previously [22]. Briefly, ${ }^{131} \mathrm{I}-\mathrm{MIP}-1095$ was prepared by iododestannylation of the trimethylstannyl precursor (S)ditert-buty 12 - ( 3 - ( ( S ) - 1 - tert - but o x y - 1 - o x o 6-(3-(4-(trimethylstannyl)phenyl)ureido)-hexane-2yl)ureido)pentanedioate, to form (S)-2-(3-((S)-1-carboxy5-(3-(4 [131I]iodophenyl)ureido)-pentyl)ureido)pentanedioic acid. [131I] NaI (approx. 7.4 GBq, GE Healthcare) was reacted with $100 \mu \mathrm{L}$ of a $250-\mu \mathrm{g} / \mathrm{mL}$ solution of (S)-di-tertb u t y 12 - ( 3 - ( ( S ) - 1 - t e r t - b u t o x y - 1 - o x o 6-(3-(4-(trimethylstannyl)phenyl)ureido)hexane-2yl)ureido)pentanedioate in ethanol and $50 \mu \mathrm{L}$ of a freshly prepared solution of $0.15 \mathrm{~mL}$ of $30 \%$ hydrogen peroxide in $0.85 \mathrm{~mL}$ of acetic acid. The reaction mixture was diluted after 5 min with $1.5 \mathrm{~mL}$ of water and loaded onto a SOLA cartridge. The cartridge was washed with $2 \mathrm{~mL}$ of water to remove unreacted radioiodide and inorganic and organic salts and dried by a stream of nitrogen. The cartridge was eluted using $500 \mu \mathrm{l}$ of neat trifluoroacetic acid (TFA) and incubated at room temperature (RT) for $7 \mathrm{~min}$. Upon dilution with $5 \mathrm{ml}$ of $\mathrm{H}_{2} \mathrm{O}$, the product was loaded onto a Bond Elut cartridge and washed with $5 \mathrm{ml}$ of $20 \%$ ethanol in water; the product was eluted using $1 \mathrm{ml}$ of ethanol, neutralized with phosphate buffer and sterile-filtered.

\section{Statistical analysis}

For statistical analysis, SigmaPlot version 12 (Systat Software Inc., Chicago, IL, USA) was used. Patients were classified into three groups (as mentioned above) according to the administered therapeutic doses. Differences between these three groups with regard to PSA response, decline of leucocytes, decline of platelets and time to PSA progression were assessed for statistical significance using Kruskal-Wallis tests. In all cases, a $p$ value of $<0.05$ was considered statistically significant.

\section{Results}

\section{Therapy efficacy}

Figure 1 represents a case of disease progression following therapy response. The best PSA responses to the RLTs are listed in Fig. 2. Different levels of PSA response following the first, second and third therapies are listed in Table 2. With regard to the whole patient cohort, mean time to PSA progression (TTP) was $116 \pm 141$ days (range 18-735; median 75) after the first therapeutic administration, $60 \pm 28$ days (range 28-117; median 50) after the second and 42 days $(n=1)$ after the third PSMA RLT with ${ }^{131}$ I-MIP-1095. Dose-related PSA response and time to progression after the endoradiotherapies are listed in Table 3 and Fig. 3.

As visible by the mentioned figure and tables, the second and third therapies were significantly less effective compared to the first therapy. There was neither an association between 

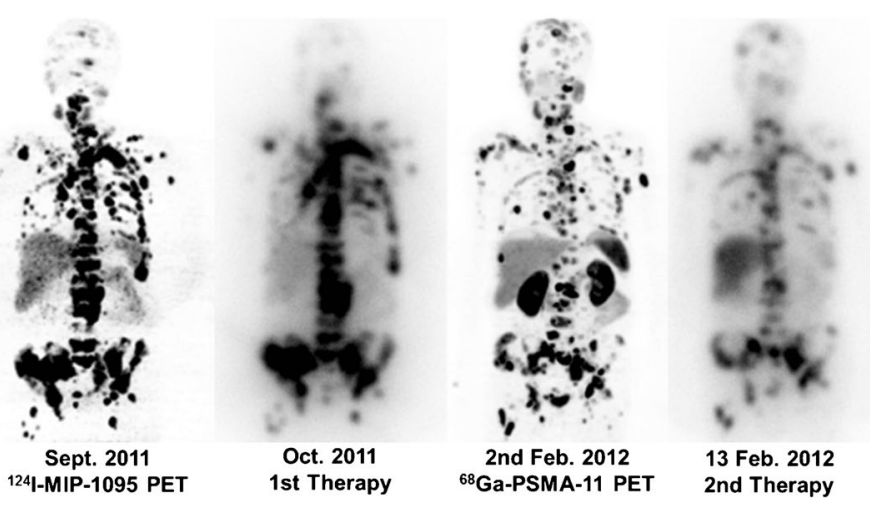

Fig. 1 Patient no. 2 received three therapies with ${ }^{131}$ I-MIP-1095. Staging before and after each therapy was conducted with PSMA ligand PET/CT $\left({ }^{124} \mathrm{I}-\mathrm{MIP}-1095\right.$ and ${ }^{68} \mathrm{Ga}$-PSMA-11). The first two therapies reduced the tumor burden. However, the third therapy didn't show a sufficient effect. The last two pictures on the right side show the rapid progress between

the applied activity and PSA response (first therapy: $p=0.70$; second therapy: $p=0.74$ ) nor between the applied activity and the time to progression (first therapy: $p=0.19$; second therapy: $p=0.61$ ).

\section{Safety and side effects}

No acute toxicity or side effects were observed in any patient during their hospitalization time (5-8 days) except one patient who reported dysgeusia after the second therapy. The dysgeusia went on for 2 weeks.

A measurable decline of blood cells within the first 10 weeks after each therapy was observed for leucocytes and platelets only (Fig. 4). However, according to the WHO CTC, no clinically relevant hematologic toxicities were observed in the majority of the patients (Table 4). CTC grade 1, 2
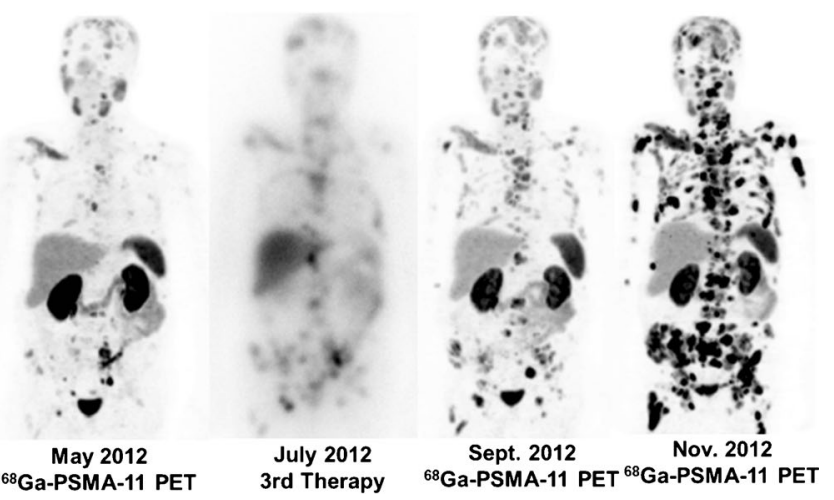

September and November. The images of the PET scans show the maximum intensity projections, those of the therapies show the geometric mean of the gamma-ray co-emission which enables imaging during therapy

and 3 hematologic toxicities were less prevalent but presented in a higher percentage of patients with increasing number of therapies (Table 4).

No significant value alterations were observed for glutamic oxaloacetic transaminase, glutamate-pyruvate transaminase, gamma-glutamyl transferase, alkaline phosphatase, bilirubin, urea, creatinine, glomerular filtration rate, thyroid-stimulating hormone, free triiodothyronine and free thyroxine (data not shown).

No association was found between the three different dose groups $(<3.5 ; 3.5-5.0$ and $>5.0 \mathrm{GBq})$ and best PSA response, decline of leucocytes, decline of platelets and time to PSA progression after the therapies. The $p$ values for the first therapy were as follows: 0.70 for best PSA response; 0.26 for decline of leucocytes, 1.00 for the decline of platelets and 0.19 for the time to PSA progression. The $p$ values for the
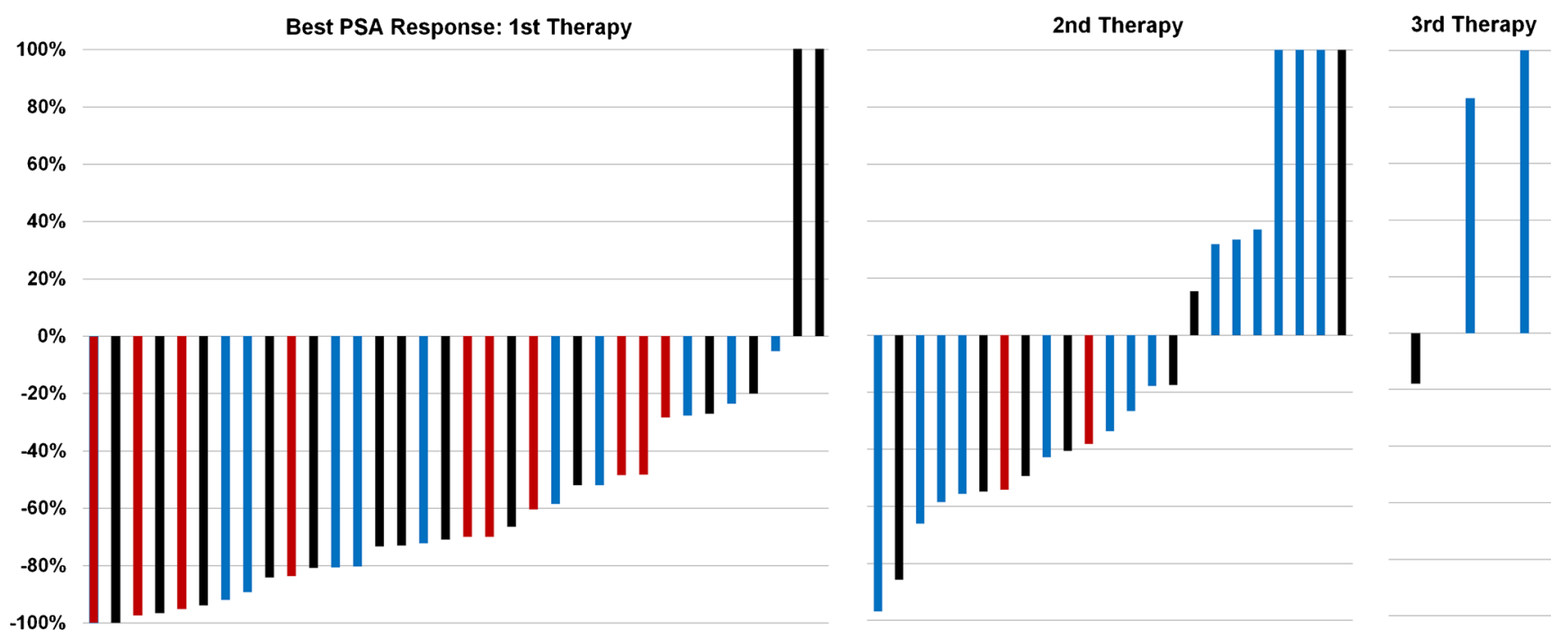

Fig. 2 Best PSA response after the first, second and third therapies with ${ }^{131}$ I-MIP-1095. As demonstrated by this figure, the second and third therapies were significantly less effective compared to the first therapy. There was no association between the applied activity and the PSA

response (first therapy: $p=0.70$; second therapy: $p=0.74$ ). Blue bars: patient group $1(<3.5 \mathrm{GBq}$ applied activity); black bars: patient group 2 (3.5-5.0 GBq applied activity); red bars: patient group 3 ( $>5 \mathrm{GBq}$ applied activity) 
Table 2 PSA responses to the first, second and third therapies with ${ }^{131}$ I-MIP-1095

\begin{tabular}{lllll}
\hline & Any PSA response & $\geq 25 \%$ PSA decline & $\geq 50 \%$ PSA decline & $\geq 75 \%$ PSA decline \\
\hline 1st therapy & $n=32(94.1 \%)$ & $n=29(85.3 \%)$ & $n=24(70.6 \%)$ & $n=13(38.2 \%)$ \\
2nd therapy & $n=15(65.2 \%)$ & $n=13(56.5 \%)$ & $n=7(30.4 \%)$ & $n=2(8.7 \%)$ \\
3rd therapy & $n=1(33.3 \%)$ & $n=0$ & $n=0$ & $n=0$ \\
\hline
\end{tabular}

second therapy were as follows: 0.74 for best PSA response; 0.17 for decline of leucocytes, 0.23 for the decline of platelets and 0.61 for the time to PSA progression. The statistical analysis could not be applied in the group with three therapies due to low number of patients $(n=3)$.

Xerostomias higher than grade 1 occurred more frequently in patients with a higher number of therapies (Table 4). The duration evaluation of this side effect was challenging. Most patients reported recovery from xerostomia after a few weeks. The duration appeared to be longer after the second or third therapy in most cases.

Dysgeusia occurred only in one patient after the second therapy (Table 4). Xerophthalmia was observed only in one patient after the second therapy. Fatigue syndromes were rare; no higher prevalence was observed with therapy numbers.

The first therapy had the strongest effect on bone pain: the majority of the patients who presented with bone pain reported a relevant reduction of the pain (reduction of analgesics or improved well-being) after the therapy. The second and third therapy led significantly less frequently to pain reduction (Table 4).

Table 3 Dose-related PSA response and time to progression following endoradiotherapy with

${ }^{131}$ I-MIP-1095

\section{Overall survival}

The median overall survival (mOS) of the patients after the first therapy with ${ }^{131}$ I-MIP-1095 was 17 months (Supplementary Table 1). At the submission of this manuscript, four patients were still alive; all of these four patients received abiraterone or enzalutamide after biochemical relapse following ${ }^{131} \mathrm{I}$-MIP-1095 therapies. The time to PSA progression of these patients was for patient 3: 64 days after the first therapy and progressive from the beginning of the second therapy; for patient 4: 649 days after the first therapy; for patient 31: 85 days after the first therapy and for patient 33: 735 days after the first therapy.

\section{Discussion}

We retrospectively report the long-term follow-up of 34 patients who received $\geq 1$ treatment with ${ }^{131}$ I-MIP1095 PSMA RLT between 2011 and 2013.

\begin{tabular}{|c|c|c|c|}
\hline & Group $1(<3.5 \mathrm{GBq})$ & Group $2(3.5-5.0 \mathrm{GBq})$ & Group 3 (>5.0 GBq) \\
\hline 1st Therapy & $(n=10)$ & $(n=14)$ & $(n=10)$ \\
\hline Best PSA response $\geq 25 \%$ & $100 \%$ & $79 \%$ & $100 \%$ \\
\hline Best PSA response $\geq 50 \%$ & $60 \%$ & $64 \%$ & $70 \%$ \\
\hline Best PSA response $\geq 75 \%$ & $40 \%$ & $36 \%$ & $40 \%$ \\
\hline Any PSA response & $100 \%$ & $86 \%$ & $100 \%$ \\
\hline TTP (PSA; days) ${ }^{*}$ & $63 \pm 24(28-90 ; 75)$ & $153 \pm 210(18-735 ; 65)$ & $91 \pm 24(28-91 ; 56)$ \\
\hline 2nd Therapy & $(n=15)$ & $(n=5)$ & $(n=3)$ \\
\hline Best PSA response $\geq 25 \%$ & $50 \%$ & $57 \%$ & $100 \%$ \\
\hline Best PSA response $\geq 50 \%$ & $29 \%$ & $57 \%$ & $50 \%$ \\
\hline Best PSA response $\geq 75 \%$ & $7 \%$ & $14 \%$ & $0 \%$ \\
\hline Any PSA response & $57 \%$ & $71 \%$ & $100 \%$ \\
\hline TTP (PSA; days) ${ }^{*}$ & $53 \pm 24(28-91 ; 50)$ & $74 \pm 41(29-117 ; 74)$ & $57 \pm 24(28-91 ; 56)$ \\
\hline 3rd Therapy & $(n=2)$ & $(n=1)$ & \\
\hline Best PSA response $\geq 25 \%$ & $0 \%$ & $100 \%$ & - \\
\hline Best PSA response $\geq 50 \%$ & $0 \%$ & $0 \%$ & - \\
\hline Best PSA response $\geq 75 \%$ & $0 \%$ & $0 \%$ & - \\
\hline Any PSA response & $0 \%$ & $100 \%$ & - \\
\hline TTP (PSA; days) ${ }^{*}$ & $42^{\sim}$ & - & - \\
\hline
\end{tabular}

* TTP: time to progression of PSA in days. $\sim$ Only one patient presented with PSA decline after the third cycle; all others presented with continuous progressive disease despite RLT 
2nd Therapy

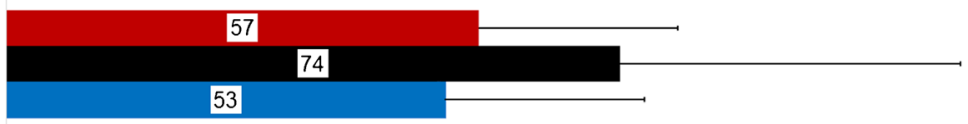

1st Therapy

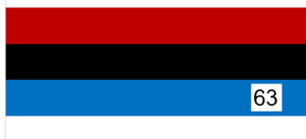

\section{1} 63

Fig. 3 Average time to PSA progression following 1-3 RLT with ${ }^{131}$ I-MIP-1095. There was no association between applied radioactivity and the time to progression (first therapy: $p=0.19$; second therapy: $p=0.61$ )

Until now - and regardless of the used radiopharmaceutical - no prospective clinical trials about PSMA RLT were initiated by pharmaceutical companies. Therefore, all experience with PSMA RLT is based on salvage therapies that were administered as an "unproven intervention in clinical practice" (Helsinki Declaration). A pre-requisite for this approach is that patients previously exhausted (or were considered "unfit" to receive) all approved therapies. Thus, in contrast to a clinical trial, inclusion criteria are not constant over time. In regard to $\mathrm{CRPC}$, the spectrum of approved
Fig. 4 Clinically relevant hematologic toxicity was observed with regard to platelets and leucocytes. W0-W10: average values of the pretherapeutical state (W0) to 10 weeks after therapy administration (W10)

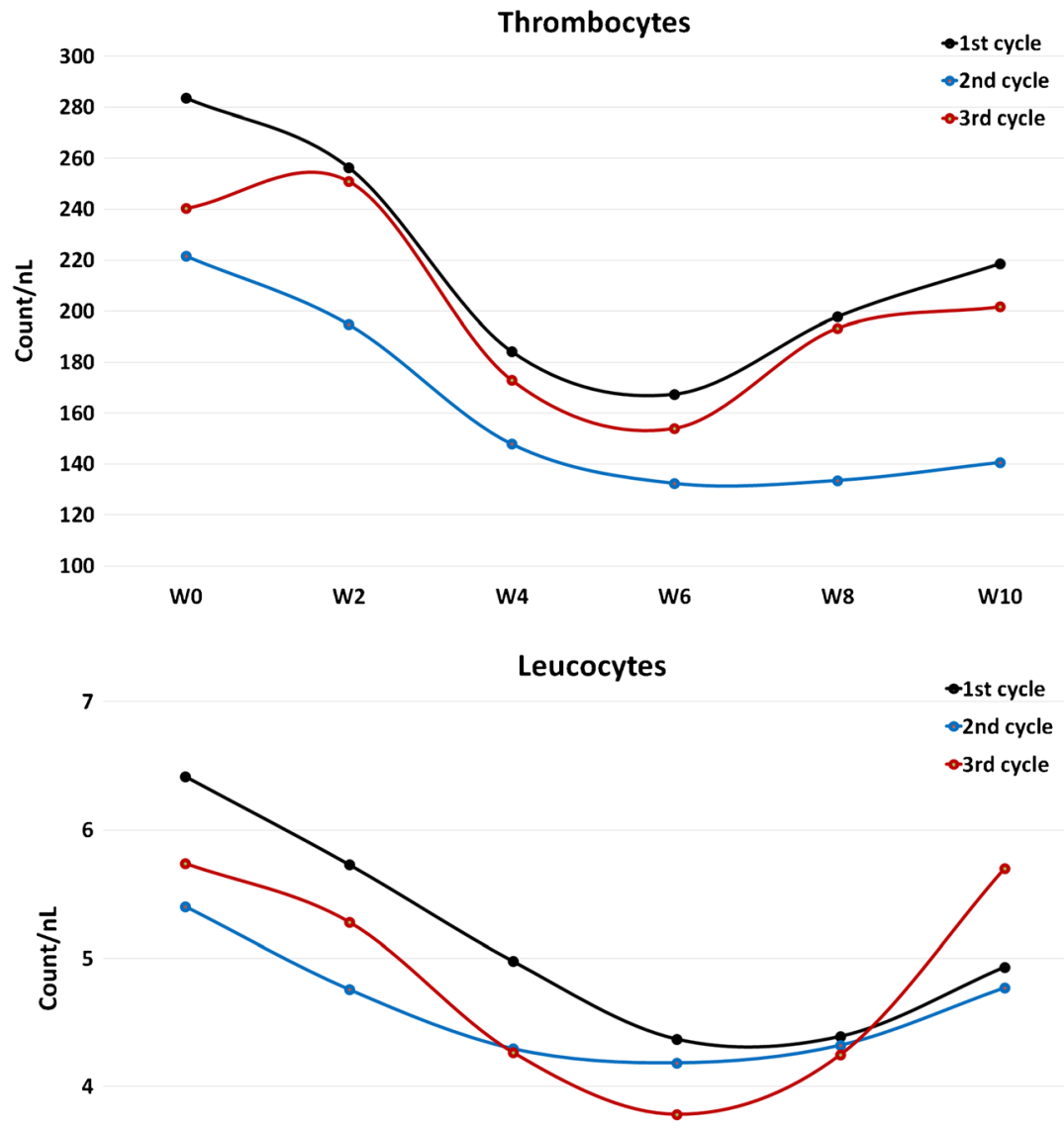

3
W2

W4
W6

w8

W10 
Table 4 Side effects and effects on bone pain of endoradiotherapy with ${ }^{131}$ I-MIP-1095

\begin{tabular}{|c|c|c|c|}
\hline & 1st Therapy & 2nd Therapy & 3rd Therapy \\
\hline Thrombopenia & No relevant toxicity in 28 patients $(82.4 \%)$ & No relevant toxicity in 15 patients $(65.2 \%)$ & No relevant toxicity in 2 patients $(66.6 \%)$ \\
\hline \multirow[t]{5}{*}{ (CTC Grade) } & Grade 1 in 3 patients $(8.58 \%)$ & Grade 1 in 2 patients $(8.7 \%)$ & - \\
\hline & Grade 2 in 1 patients $(2.9 \%)$ & Grade 2 in 3 patients (13.0\%) & - \\
\hline & Grade 3 in 2 patients $(5.9 \%)$ & Grade 3 in 2 patients $(8.7 \%)$ & - \\
\hline & - & - & Grade 4 in 1 patient $(33.3 \%)$ \\
\hline & - & Grade $1 \rightarrow$ Grade 3 in 1 patient $(4.3 \%)$ & - \\
\hline \multirow[t]{5}{*}{ Leukopenia } & No relevant toxicity in 20 patients $(58.8 \%)$ & No relevant toxicity in 11 patients $(47.8 \%)$ & No relevant toxicity in 1 patient $(33.3 \%)$ \\
\hline & Grade 1 in 8 patients $(23.5 \%)$ & Grade 1 in 6 patients $(26.0 \%)$ & Grade 1 in 1 patient $(33.3 \%)$ \\
\hline & Grade 2 in 5 patients $(14.7 \%)$ & Grade 2 in 4 patients $(17.4 \%)$ & Grade 2 in 1 patient $(33.3 \%)$ \\
\hline & Grade 3 in 1 patient $(2.9 \%)$ & - & - \\
\hline & - & Grade $1 \rightarrow$ grade 2 in 2 patient $(8.7 \%)$ & - \\
\hline \multirow[t]{4}{*}{ Xerostonia } & Grade 0 in 4 patients $(11.8 \%)$ & Grade 0 in 0 patient $(0.0 \%)$ & Grade 0 in 0 patients $(0.0 \%)$ \\
\hline & Grade 1 in 24 patients $(70.6 \%)$ & Grade 1 in 14 patients $(60.9 \%)$ & Grade 1 in 2 patients $(66.6 \%)$ \\
\hline & Grade 2 in 6 patients $(17.6 \%)$ & Grade 2 in 6 patients $(26.1 \%)$ & Grade 2 in 0 patients $(0.0 \%)$ \\
\hline & Grade 3 in 0 patients $(0.0 \%)$ & Grade 3 in 3 patients $(13.0 \%)$ & Grade 3 in 1 patient $(33.3 \%)$ \\
\hline Dysgeusia & None & 1 Patient $(4.3 \%)$ & None \\
\hline Xerophthalmia & None & 1 Patient $(4.3 \%)$ & None \\
\hline Fatigue & 2 Patients $(5.9 \%)$ & 2 Patients $(8.7 \%)$ & 1 Patient $(33.3 \%)$ \\
\hline \multirow[t]{3}{*}{ Bone pain } & Pain prior to therapy: 16 patients $(47 \%)$ & Pain prior to therapy: 11 patients $(47.8 \%)$ & Pain prior to therapy: 2 patients $(66.6 \%)$ \\
\hline & $\begin{array}{l}\text { Reduction after therapy: } 15 \text { patients } \\
(93.8 \%)\end{array}$ & Reduction after therapy: 3 patients $(27.3 \%)$ & Reduction after therapy: 0 patients $(0 \%)$ \\
\hline & $\begin{array}{l}\text { Uncharged intensity after therapy: } 1 \\
\text { patient }(6.2 \%)\end{array}$ & $\begin{array}{l}\text { Uncharged intensity after therapy: } 8 \text { patients } \\
(72.7 \%)\end{array}$ & $\begin{array}{l}\text { Uncharged intensity after therapy: } 2 \text { patients } \\
(100 \%)\end{array}$ \\
\hline
\end{tabular}

pharmaceuticals changed dramatically during the reported inclusion period.

In Europe, abiraterone was approved for patients pre-treated with docetaxel in 09/2011 and before chemotherapy in 01/2013. Enzalutamide was first approved 06/2013 to be used after docetaxel and in 12/2014 to be used before docetaxel. Cabazitaxel is approved as a second-line chemotherapy only, since 2011. In 12/ $2012{ }^{223} \mathrm{RaCl}$ was approved for a subgroup of patients with bone-confined tumor spread. Dependent on the actual approved indication, practical availability and the patient's appropriateness to receive chemotherapy, which was classified by the responsible uro-/oncologist independently from the nuclear medicine physician, alternative options were given priority even if patients presented with a good response to the previous PSMA RLT. This explains the low number of 2 nd and 3rd therapies, despite $>70 \%$ of patients having a decline of $>50 \%$ in serum PSA and an mTTP of 116 days after the first treatment.

Nevertheless, despite being heterogeneous, these data are still of particular interest because they present the only experience with PSMA RLT in the pre-abiraterone, pre-enzalutamid, pre- ${ }^{223} \mathrm{RaCl}$ era. In contrast, reports in recent publications about ${ }^{177}$ Lu-labeled PSMA RLT have been for patients after secondary hormone manipulation [13, 14, 20, 23-25]. There is growing experience about cross-resistance between abiraterone and enzalutamide [26] as both drugs are targeting the androgen receptor (AR) axis. If it would be possible to keep these treatment lines apart by interleaving non-AR-targeting therapies such as PSMA RLT, the interruption of selection pressure eventually might reverse cross-resistance. One case report about restored hormone response after ${ }^{177} \mathrm{Lu}-\mathrm{PSMA}-$ 617 generates a promising thesis [27]. In our collective, there are four still-living patients, which were successfully bridged by ${ }^{131}$ I-MIP1095 RLTs until novel options became available and who now present with enduring responses to the succeeding secondary hormone manipulations.

In contrast to the before-mentioned publications about PSMA RLT [13, 14, 20, 23-25], in our cohort, the succeeding application of the radiopharmaceutical was delayed until progression of disease. One observation is that the mTTP after each further treatment decreases. Treatment regimens administered in cycles of fixed intervals shorter than the here-observed mTTP of 75 days (2.5 months) might be one option to prolong progression-free survival. In 2013, our department switched to PSMA-RLT intervals of every two months and the mTTP doubled to about 5 months [14] which is also in accordance to the prolonged PFS reported by other groups $[13,20]$. Nevertheless, 
it remains unclear whether a prolonged mPFS or mTTP achieved by intensified treatment regimens will finally also translate into a longer mOS.

Based on the dosimetry estimates with ${ }^{124}$ I-MIP1095 PET and extrapolation to ${ }^{131}$ I-MIP1095 RLT [22], the mean absorbed doses to salivary glands are $4.62 \mathrm{~Gy} / \mathrm{GBq}$ and to the red marrow $0.31 \mathrm{~Gy} / \mathrm{GBq}$. A therapy with $4 \mathrm{GBq}{ }^{131} \mathrm{I}$ MIP1095, therefore, corresponds to an 18.5-Gy salivary gland dose and a 1.2-Gy red marrow dose. A red marrow dose of 2 Gy is considered safe for RLT and, therefore, the herereported low number of grade 3 hematological toxicities $(6 \%)$ after the first therapy are within the expectations. Nevertheless, as demonstrated by Fig. 4, a moderate depression of blood cell count was observed after each cycle with the nadir around 6 weeks post-therapy. PSMA RLT with the radiolabeled antibody ${ }^{177} \mathrm{Lu}-\mathrm{J} 591$ resulted in comparable red marrow doses [9] and hematological toxicity as well as the platelet nadir are also in accordance with our results [28]. In contrast, with ${ }^{177} \mathrm{Lu}$-PSMA-617, the red marrow dose is about $0.025-0.03 \mathrm{~Gy} / \mathrm{GBq}[14,29]$ and therapy with $6 \mathrm{GBq}{ }^{177} \mathrm{Lu}-$ PSMA-617 translates into an estimated red marrow dose of $0.15-0.2$ Gy. Thus, the even better tolerability of this radioconjugate regarding hematological toxicity is reasonable.

With ${ }^{177} \mathrm{Lu}-\mathrm{PSMA}-617,1.4 \mathrm{~Gy} / \mathrm{GBq}$ (e.g. $8.4 \mathrm{~Gy}$ for $6 \mathrm{GBq}$ ) has been calculated as the salivary gland's absorbed dose [29]. Thus, it is plausible that we find a higher incidence of moderate xerostomia with our treatment regimen than it is reported for ${ }^{177}$ Lu-PSMA RLT [13, 14, 20, 23-25]. However, with ${ }^{131} \mathrm{I}-$ MIP-1095, we also found a higher rate of responders in regard to both "any PSA decline" (94\%) and " $>50 \%$ PSA decline" (71\%). In the largest report $(n=82)$ about ${ }^{177}$ Lu-PSMA-617 administered in fractions of $6 \mathrm{GBq}$ [30], the corresponding response rates for "any PSA decline" $(64 \%)$ and " $>50 \%$ PSA decline" (31\%) were remarkably lower. As the variability of absorbed dose to different tumor lesions is high, no reliable comparison between the two ligands regard to their therapeutic range is possible. We would emphasize that dose escalation of ${ }^{177}$ Lu-PSMA-617 will increase both response rate and toxicity. Alternatively, de-escalation of ${ }^{131}$ I-MIP-1095 seems also a reasonable concept because there was no relevant difference in response rate between patients that received $<3.5$ or $>5 \mathrm{GBq}$ ${ }^{131}$ I-MIP-1095 (Table 3). As stated above, the analysis of this paper was done during the pre-abiraterone and pre-enzalutamide era which is in contrast to the newer reports with ${ }^{177}$ Lu-PSMA617. Clinical studies revealed an increase of PSMA expression in tissue specimens after androgen-deprivation therapy [31,32]. At present, no data are available concerning a further increase or a decrease of PSMA expression after abiraterone and/or enzalutamide. Therefore, application of ${ }^{131}$ I-MIP-1095 in a similar clinical setting may deliver a better data set for comparison. On the other hand, it is known that the later a therapy line is applied, the lower the efficacy due to an increase of tumor aggressiveness [33, 34].
One reason that more centers currently rely on ${ }^{177} \mathrm{Lu}$ labeled PSMA ligands is the lower co-emission of gamma radiation. ${ }^{131} \mathrm{I}$ has an $82 \%$ abundance probability for highenergetic $(364 \mathrm{keV}) \gamma$-radiation and an $89 \%$ abundance probability for $\beta$-radiation $(606 \mathrm{keV})$. In contrast, ${ }^{177} \mathrm{Lu}$ has a $\gamma$ co-emission of only $11 \%(210 \mathrm{keV})$ but a $100 \%$ abundance probability for $\beta$-radiation $(490 \mathrm{keV})$. Depending on radiation protection acts, this can translate into different needs for isolation. Our patients who were treated with ${ }^{131}$ I-MIP-1095 had an average hospitalization time of 7 days while those treated with ${ }^{177}$ Lu-PSMA- 617 were hospitalized for 2 days. A second reason might be the level of efforts for radiolabeling. Chelator-containing ligands can be labeled with high and robust labeling yields, the synthesis of ${ }^{131}$ I-MIP-1095 on-site were one reason for the high variance of treatment activity. However, this might easily be overcome by a routine production by commercial companies.

\section{Conclusion}

The first clinical experience with therapeutic small-molecule PSMA ligands was using ${ }^{131}$ I-MIP-1095 as a single-cycle therapy. The here-reported experience of repeated applications of PSMA RLT after the next PSA relapse is still unique. The deepness of PSA response and duration of tumor control was most pronounced with the first therapy but repeated treatments were already facing resistance. Repeated full-dose therapy also causes more side effects such as hematological toxicity and xerostomia. This provides a rationale for metronomic or fractionated treatment regimens. The first experiences based on fractionated therapy with ${ }^{177}$ Lu-PSMA ligands have recently been published. However, it has yet to be proven which of these concepts will be superior with regard to overall survival.

Acknowledgements We express our deepest gratitude to all patients' relatives who provided valuable information for this study despite being confronted with painful memories.

We also express our gratitude to Mrs. Gisela Schlesinger-Woweries and Mrs. Tanja Gerber for their help in collecting a significant part of the data.

\section{Compliance with ethical standards}

Conflicts of interest Molecular Insight Pharmaceuticals, Inc. (a wholly-owned subsidiary of Progenics Pharmaceuticals), New York City, NY, USA had a role in sponsoring material used in the study. Thomas Armor is a full-time employee of Progenics Pharmaceuticals, Inc. All other authors declare that they have no conflicts of interest.

Ethical approval All patients published in this manuscript signed a written informed consent form for the purpose of anonymized evaluation and publication of their data. All reported investigations were conducted in accordance with the Helsinki Declaration and with our national regulations (German Medicinal Products Act, AMG $\S 132 b$ ). This evaluation was approved by the ethics committee of the University of Heidelberg (S-321-2012). 
Open Access This article is distributed under the terms of the Creative Commons Attribution 4.0 International License (http:// creativecommons.org/licenses/by/4.0/), which permits unrestricted use, distribution, and reproduction in any medium, provided you give appropriate credit to the original author(s) and the source, provide a link to the Creative Commons license, and indicate if changes were made.

\section{References}

1. Vallabhajosula S, Nikolopoulou A, Babich JW, Osborne JR, Tagawa ST, Lipai I, et al. 99mTc-labeled small-molecule inhibitors of prostate-specific membrane antigen: pharmacokinetics and biodistribution studies in healthy subjects and patients with metastatic prostate cancer. J Nucl Med Off Publ Soc Nucl Med. 2014;55: 1791-8.

2. Szabo Z, Mena E, Rowe SP, Plyku D, Nidal R, Eisenberger MA, et al. Initial evaluation of [(18)F]DCFPyL for Prostate-Specific Membrane Antigen (PSMA)-targeted PET imaging of prostate cancer. Mol Imaging Biol Off Publ Acad Mol Imaging. 2015;17:565-74.

3. Afshar-Oromieh A, Avtzi E, Giesel FL, Holland-Letz T, Linhart $\mathrm{HG}$, Eder M, et al. The diagnostic value of PET/CT imaging with the (68)Ga-labelled PSMA ligand HBED-CC in the diagnosis of recurrent prostate cancer. Eur J Nucl Med Mol Imaging. 2015;42: 197-209.

4. Kratochwil C, Afshar-Oromieh A, Kopka K, Haberkorn U, Giesel FL. Current status of prostate-specific membrane antigen targeting in nuclear medicine: clinical translation of chelator containing prostate-specific membrane antigen ligands into diagnostics and therapy for prostate cancer. Semin Nucl Med. 2016;46:405-18.

5. Afshar-Oromieh A, Babich JW, Kratochwil C, Giesel FL, Eisenhut $\mathrm{M}$, Kopka K, et al. The rise of PSMA ligands for diagnosis and therapy of prostate cancer. J Nucl Med Off Publ Soc Nucl Med. 2016;57:79S-89S

6. Barrett JA, Coleman RE, Goldsmith SJ, Vallabhajosula S, Petry NA, Cho S, et al. First-in-man evaluation of 2 high-affinity PSMA-avid small molecules for imaging prostate cancer. J Nucl Med Off Publ Soc Nucl Med. 2013;54:380-7.

7. Hillier SM, Maresca KP, Femia FJ, Marquis JC, Foss CA, Nguyen $\mathrm{N}$, et al. Preclinical evaluation of novel glutamate-urea-lysine analogues that target prostate-specific membrane antigen as molecular imaging pharmaceuticals for prostate cancer. Cancer Res. 2009;69: 6932-40.

8. Haberkorn U, Eder M, Kopka K, Babich JW, Eisenhut M. New strategies in prostate cancer: Prostate-Specific Membrane Antigen (PSMA) ligands for diagnosis and therapy. Clin Cancer Res Off $\mathrm{J}$ Am Assoc Cancer Res. 2016;22:9-15.

9. Vallabhajosula S, Goldsmith SJ, Hamacher KA, Kostakoglu L, Konishi S, Milowski MI, et al. Prediction of myelotoxicity based on bone marrow radiation-absorbed dose: radioimmunotherapy studies using 90Y- and 177Lu-labeled J591 antibodies specific for prostate-specific membrane antigen. J Nucl Med Off Publ Soc Nucl Med. 2005;46:850-8.

10. Milowsky MI, Nanus DM, Kostakoglu L, Vallabhajosula S, Goldsmith SJ, Bander NH. Phase I trial of yttrium-90-labeled anti-prostate-specific membrane antigen monoclonal antibody J591 for androgen-independent prostate cancer. J Clin Oncol. 2004;22: 2522-31.

11. Tagawa ST, Milowsky MI, Morris M, Vallabhajosula S, Christos P, Akhtar NH, et al. Phase II study of lutetium-177-labeled anti-prostate-specific membrane antigen monoclonal antibody J591 for metastatic castration-resistant prostate cancer. Clin Cancer Res Off J Am Assoc Cancer Res. 2013;19:5182-91.
12. Ahmadzadehfar H, Rahbar K, Kürpig S, Bögemann M, Claesener M, Eppard E, et al. Early side effects and first results of radioligand therapy with (177)Lu-DKFZ-617 PSMA of castrate-resistant metastatic prostate cancer: a two-centre study. EJNMMI Res. 2015;5:114.

13. Baum RP, Kulkarni HR, Schuchardt C, Singh A, Wirtz M, Wiessalla S, et al. 177Lu-labeled prostate-specific membrane antigen radioligand therapy of metastatic castration-resistant prostate cancer: safety and efficacy. J Nucl Med Off Publ Soc Nucl Med. 2016;57:1006-13.

14. Kratochwil C, Giesel FL, Stefanova M, Benešová M, Bronzel M, Afshar-Oromieh A, et al. PSMA-targeted radionuclide therapy of metastatic castration-resistant prostate cancer with 177Lu-labeled PSMA-617. J Nucl Med Off Publ Soc Nucl Med. 2016;57:1170-6.

15. Rahbar K, Ahmadzadehfar H, Kratochwil C, Haberkorn U, Schäfers M, Essler M, et al. German multicenter study investigating $177 \mathrm{Lu}$-PSMA-617 radioligand therapy in advanced prostate cancer patients. J Nucl Med Off Publ Soc Nucl Med. 2016.

16. Fendler WP, Reinhardt S, Ilhan H, Delker A, Böning G, Gildehaus FJ, et al. Preliminary experience with dosimetry, response and patient reported outcome after 177Lu-PSMA617 therapy for metastatic castration-resistant prostate cancer. Oncotarget. 2016

17. Ferdinandus J, Eppard E, Gärtner F, Kürpig S, Fimmers R, Yordanova A, et al. Predictors of response to radioligand therapy of metastatic castrate-resistant prostate cancer with 177Lu-PSMA617. J. Nucl Med Off Publ Soc Nucl Med. 2016.

18. Yadav MP, Ballal S, Tripathi M, Damle NA, Sahoo RK, Seth A, et al. (177)Lu-DKFZ-PSMA-617 therapy in metastatic castration resistant prostate cancer: safety, efficacy, and quality of life assessment. Eur J Nucl Med Mol Imaging. 2016

19. Kratochwil C, Bruchertseifer F, Giesel FL, Weis M, Verburg FA, Mottaghy F, et al. 225Ac-PSMA-617 for PSMA targeting alpharadiation therapy of patients with metastatic castration-resistant prostate cancer. J Nucl Med Off Publ Soc Nucl Med. 2016

20. Heck MM, Retz M, D'Alessandria C, Rauscher I, Scheidhauer K, Maurer T, et al. Systemic radioligand therapy with (177)Lu labeled prostate specific membrane antigen ligand for imaging and therapy in patients with metastatic castration resistant prostate cancer. $\mathrm{J}$ Urol. 2016;196:382-91.

21. Rahbar K, Bode A, Weckesser M, Avramovic N, Claesener M, Stegger L, et al. Radioligand therapy with 177Lu-PSMA-617 as a novel therapeutic option in patients with metastatic castration resistant prostate cancer. Clin Nucl Med. 2016;41:522-8.

22. Zechmann CM, Afshar-Oromieh A, Armor T, Stubbs JB, Mier W, Hadaschik B, et al. Radiation dosimetry and first therapy results with a (124)I/ (131)I-labeled small molecule (MIP-1095) targeting PSMA for prostate cancer therapy. Eur J Nucl Med Mol Imaging. 2014;41:1280-92.

23. Ahmadzadehfar H, Essler M, Schäfers M, Rahbar K. Radioligand therapy with (177)Lu-PSMA-617 of metastatic prostate cancer has already been arrived in clinical use. Nucl Med Biol 2016.

24. Rahbar K, Bögemann M, Ahmadzadehfar H. (177)Lu-PSMA-617 radioligand therapy of $\mathrm{mCRPC}$ : evaluation criteria of response. Eur J Nucl Med Mol Imaging. 2016.

25. Fendler WP, Reinhardt S, Ilhan H, Delker A, Böning G, Gildehaus FJ, et al. Preliminary experience with dosimetry, response and patient reported outcome after 177Lu-PSMA-617 therapy for metastatic castration-resistant prostate cancer. Oncotarget. 2016.

26. van Soest RJ, van Royen ME, de Morrée ES, Moll JM, Teubel W, Wiemer EA, et al. Cross-resistance between taxanes and new hormonal agents abiraterone and enzalutamide may affect drug sequence choices in metastatic castration-resistant prostate cancer. Eur J Cancer Oxf Engl. 2013;49:3821-30. 
27. Schlenkhoff CD, Knüpfer E, Essler M, Ahmadzadehfar H. Metastatic prostate cancer with restored hormone-response after radioligand therapy with 177Lu-PSMA-617. Clin Nucl Med. 2016;41:572-3.

28. Vallabhajosula S, Nikolopoulou A, Jhanwar YS, Kaur G, Tagawa ST, Nanus DM, et al. Radioimmunotherapy of metastatic prostate cancer with 177Lu-DOTAhuJ591 anti prostate specific membrane antigen specific monoclonal antibody. Curr Radiopharm. 2016;9: 44-53.

29. Delker A, Fendler WP, Kratochwil C, Brunegraf A, Gosewisch A, Gildehaus FJ, et al. Dosimetry for (177)LuDKFZ-PSMA-617: a new radiopharmaceutical for the treatment of metastatic prostate cancer. Eur J Nucl Med Mol Imaging. 2016;43:42-51.

30. Rahbar K, Schmidt M, Heinzel A, Eppard E, Bode A, Yordanova A, et al. Response and tolerability of a single dose of $177 \mathrm{Lu}-$ PSMA-617 in patients with metastatic castration-resistant prostate cancer: a multicenter retrospective analysis. J Nucl Med Off Publ Soc Nucl Med. 2016;57:1334-8.
31. Hope TA, Truillet CC, Ehman EC, Afshar-Oromieh A, Aggarwal $\mathrm{R}$, Ryan CJ, et al. Imaging response to androgen receptor inhibition using 68Ga-PSMA-11 PET: first human experience. J Nucl Med Off Publ Soc Nucl Med. 2016.

32. Wright Jr GL, Grob BM, Haley C, Grossman K, Newhall K, Petrylak D, et al. Upregulation of prostate-specific membrane antigen after androgen-deprivation therapy. Urology. 1996;48: 326-34.

33. Fizazi K, Scher HI, Molina A, Logothetis CJ, Chi KN, Jones RJ, et al. Abiraterone acetate for treatment of metastatic castrationresistant prostate cancer: final overall survival analysis of the COU-AA-301 randomised, double-blind, placebo-controlled phase 3 study. Lancet Oncol. 2012;13:983-92.

34. Ryan CJ, Smith MR, Fizazi K, Saad F, Mulders PFA, Sternberg CN, et al. Abiraterone acetate plus prednisone versus placebo plus prednisone in chemotherapy-naive men with metastatic castrationresistant prostate cancer (COU-AA-302): final overall survival analysis of a randomised, double-blind, placebo-controlled phase 3 study. Lancet Oncol. 2015;16:152-60. 